\title{
Coulomb gauge on the lattice: From zero to finite temperature
}

\author{
Hannes Vogt*, Giuseppe Burgio, Markus Quandt, Hugo Reinhardt \\ Eberhard Karls Universität Tübingen, Institut für Theoretische Physik \\ E-mail: \\ hannes.vogteuni-tuebingen.de, giuseppe.burgio@uni-tuebingen.de, \\ markus.quandtauni-tuebingen. de, hugo.reinhardteuni-tuebingen. de
}

Our previous studies of Coulomb gauge Yang-Mills theory are extended to finite temperature. We investigate the SU(2) static gluon and ghost propagators and show results for the Coulomb potential, with a focus on the Gribov ambiguity. To compute these quantities at high temperatures and to solve scaling violations we use the anisotropic Wilson gauge action.

31st International Symposium on Lattice Field Theory LATTICE 2013

July 29 - August 3, 2013

Mainz, Germany

\footnotetext{
* Speaker.
} 


\section{Introduction}

Zero temperature QCD in Coulomb gauge was subject of extensive studies both in the continuum formulation [1,2] and on the lattice $[3,4,5,6,7,8,9,10,11,12]$. A summary of our findings was given in the talk of G. Burgio at this conference [13]. Recently, finite temperature was investigated in the Hamiltonian variational approach [14] where they found a clear signal of the deconfinement phase transition in the infrared exponent of the ghost propagator. In the following we will extend our lattice calculations to finite temperature, in search for a manifestation of the deconfinement phase transition in Coulomb gauge correlation functions.

\section{Lattice setup}

In our study we use the anisotropic $\mathrm{SU}(2)$ Wilson gauge action which allows us to simulate a large variety of temperatures (up to $6 T_{C}$ ) at still large lattice volumes. Additionally, scaling violations in the Coulomb gauge propagators have been shown to get milder for large anisotropies and are expected to vanish in the lattice Hamiltonian limit $(\xi \rightarrow \infty)[12,11]$. The action is defined by

$$
\begin{aligned}
S[U] & =\sum_{n \in \Lambda}\left\{\frac{\beta_{s}}{N_{c}} \sum_{i=1}^{3} \sum_{j=i+1}^{3} \operatorname{Retr}\left[\mathbb{1}-U_{i j}(n)\right]+\frac{\beta_{t}}{N_{c}} \sum_{i=1}^{3} \operatorname{Retr}\left[\mathbb{1}-U_{0 i}(n)\right]\right\} \\
& =\frac{\beta}{N_{c}} \sum_{n \in \Lambda}\left\{\frac{1}{\xi_{0}} \sum_{i=1}^{3} \sum_{j=i+1}^{3} \operatorname{Re} \operatorname{tr}\left[\mathbb{1}-U_{i j}(n)\right]+\xi_{0} \sum_{i=1}^{3} \operatorname{Retr}\left[\mathbb{1}-U_{0 i}(n)\right]\right\}
\end{aligned}
$$

with the inverse coupling $\beta_{s}=\beta / \xi_{0}$ in spatial directions and $\beta_{t}=\beta \xi_{0}$ in the temporal direction. We adjusted the bare anisotropy $\xi_{0}$ to correspond to a renormalized anisotropy $\xi=a_{s} / a_{t}=4$ using the data from [12]. For scale setting we assume a Wilson string tension of $\sigma=(440 \mathrm{MeV})^{2}$.

We used lattices of size $N_{t} \times 32^{3}$ where $N_{t}=128$ for $T=0$ and $N_{t} \in[4,32]$ for $T>T_{C}$. For each dataset 100 configurations where generated with heatbath and overrelaxation updates.

Coulomb gauge on the lattice is implemented by maximizing the functional

$$
F^{g}[U](t)=\frac{1}{4 N_{c} N_{s}^{3}} \operatorname{Re} \sum_{x, i} \operatorname{tr}\left[g(\vec{x}, t) U_{i}(\vec{x}, t) g(\vec{x}+\hat{\imath}, t)^{\dagger}\right]
$$

in each timeslice with respect to local gauge transformations $g(x) \in \mathrm{SU}(2)$. Each local maximum satisfies the Coulomb gauge condition $\partial_{i} A_{i}=0$. It is well known, that (1.1) has very many local maxima which leads to the so-called Gribov problem [15]. To avoid the Gribov problem we try to find the global maximum of (1.1) by using the simulated annealing algorithm [16] and multiple restarts of the gauge fixing procedure on random gauge transformations. For the numerical optimization we use a SU(2) implementation of the simulated annealing and overrelaxation algorithm on GPUs [17].

The Coulomb gauge condition is not complete: a residual space-independent gauge freedom is left unfixed. We remove this residual gauge freedom by the integrated Polyakov gauge defined in [8], which is a lattice version of $\partial_{0} \int \mathrm{d}^{3} \vec{x} A_{0}(\vec{x}, t)=0$. Residual gauge fixing does not affect the ghost propagator and the Coulomb potential, since these quantities are defined at fixed time $t$. In each timeslice $t$, Coulomb gauge fixing is complete and any space-independent gauge transformation is just a global transformation. 


\section{Gluon propagator}

In this study, we decided to use the definition of the static gluon propagator introduced in [8]. There, a factorization of the full propagator in a function of $|\vec{p}|$ and a function of $|\vec{p}| / p_{0}$ is found and used to eliminate the energy dependence. On anisotropic lattices this procedure still works, but deviations from the factorization are found. Currently, we are investigating if this effect is only due to the larger uncertainties in the scale setting on the anisotropic lattices or if the simple averaging used in [11] should be preferred. ${ }^{1}$

In [8], the SU(2) gluon propagator on isotropic lattices was found to satisfy the Gribov formula

$$
D(|\vec{p}|)=\frac{1}{\sqrt{|\vec{p}|^{2}+\frac{M^{4}}{|\vec{p}|^{2}}}} .
$$

This simple ansatz does not work anymore for the propagator on anisotropic lattices. If we want to keep to a similar form we need to add additional parameters

$$
\frac{D(|\vec{p}|)}{|\vec{p}|}=\frac{1}{\sqrt{|\vec{p}|^{4}+\gamma M^{2}|\vec{p}|^{2}+\alpha M^{3}|\vec{p}|+M^{4}}}
$$

where we divided by $|\vec{p}|$ to get a clearer look at the IR behaviour: a non-diverging value at $|\vec{p}| \rightarrow 0$ means a vanishing propagator.

(a)

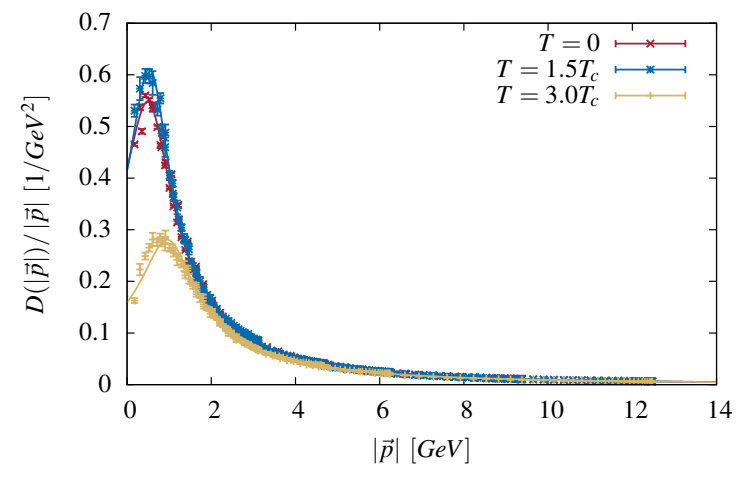

(b)

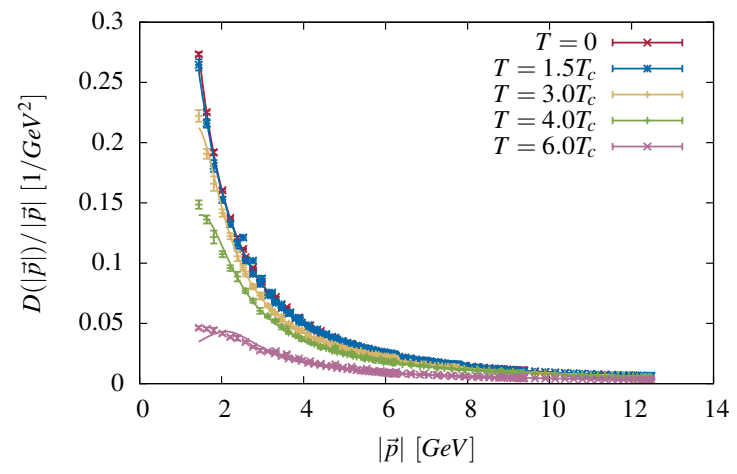

Figure 1: $D(|\vec{p}|) /|\vec{p}|$ (a) for $T=0,1.5 T_{C}$ and $3 T_{C}$ with 5 sets with $\beta \in[2.25,2.64]$ at each temperature. (b) up to $6 T_{C}$ with 3 or 4 sets with $\beta \in[2.49,2.64]$

In Fig. 1(a) we show $D(|\vec{p}|) /|\vec{p}|$ at $T=0,1.5 T_{C}$ and $3 T_{C}$ for 5 sets of configurations with the same parameters $\beta$ and $\xi_{0}$ at each temperature. To avoid spurious effects of the fitting procedure between different $T$ we did a combined fit to (2.2) with 14 parameters: $M, \alpha$ and $\gamma$ for each $T$ and a renormalization constant for each $\left(\beta, \xi_{0}\right)$. The propagator at zero temperature and at $1.5 T_{C}$ does not show a clear difference. We expect that the small increase around the peak would go away at weaker coupling and with better statistics. This is also indicated by the propagator at $3 T_{C}$, where a distinct difference is now visible, but the propagator is now below the zero temperature propagator. This behaviour goes on towards higher temperatures (at least up to $6 T_{C}$ ), see Fig. 1(b).

\footnotetext{
${ }^{1}$ The following qualitative statements about the finite temperature behaviour of the propagator is not affected by the definition.
} 


\section{Ghost dressing function}

The ghost propagator in momentum space is given by

$$
G(|\vec{p}|)=\frac{d(|\vec{p}|)}{|\vec{p}|^{2}}=\frac{\delta^{a b}}{N_{s}^{3}\left(N_{c}^{2}-1\right)}\left\langle\sum_{\vec{x}, \vec{y}} \mathrm{e}^{\mathrm{i} \vec{p}(\vec{x}-\vec{y})}\left[M^{-1}\right]^{a b}(\vec{x}, \vec{y})\right\rangle
$$

where $M$ is the discrete Faddeev-Popov operator in Coulomb gauge. The ghost form factor $d(|\vec{p}|)$ is found to behave as a power $\kappa$ of the momentum $|\vec{p}|$ in the infrared and has logarithmic corrections with anomalous dimension $\gamma$ in the ultraviolet regime:

$$
d(|\vec{p}|) \sim \frac{1}{|\vec{p}|^{\kappa}} \quad d(|\vec{p}|) \sim \frac{1}{\log ^{\gamma}\left(\frac{|\vec{p}|}{m}\right)} .
$$

In [12] an infrared exponent of $\kappa \approx 0.5$ was found by extrapolating to the Hamiltonian limit $(\xi \rightarrow \infty)$ for the zero temperature case. This is in disagreement with findings in the Hamiltonian variational approach which favor $\kappa=1$ [2]. See also [13].

(a)

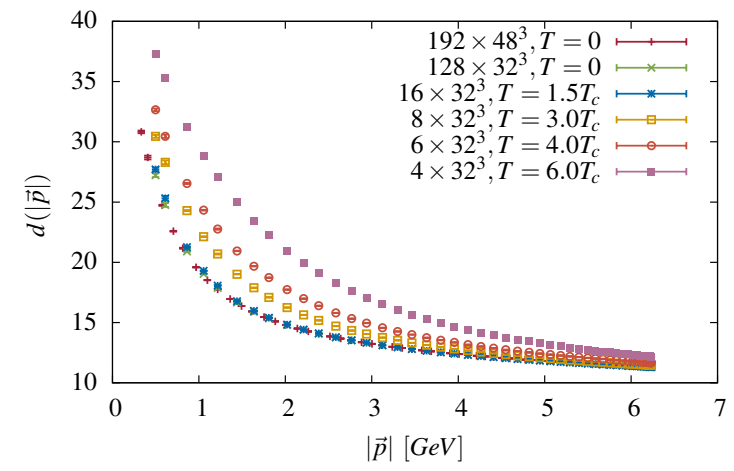

(b)

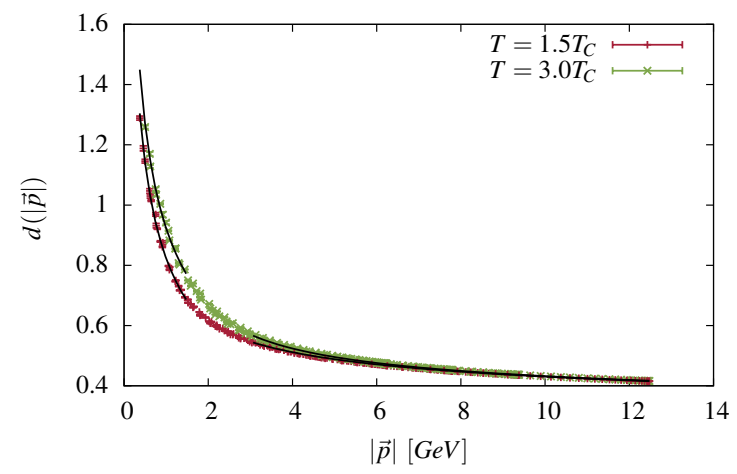

Figure 2: The ghost dressing function $d(|\vec{p}|)$ : (a) for fixed $\beta=2.5$. (b) for $T=1.5 T_{C}$ and $3.0 T_{C}$ from 4 or 5 sets of $\beta \in[2.40,2.64]$.

In Fig. 2(a) we show the ghost dressing function for fixed $\beta$. Finite volume effects are under control in the $128 \times 32^{3}$ lattices as indicated by the comparison with the $192 \times 48^{3}$ data. As in the case of the gluon, the ghost propagator is not sensitive to the deconfinement phase transition: we started with an IR exponent of about a half at $T=0$ and still have the same exponent above $T_{C}$. Again, starting from the $3 T_{C}$ data deviations are visible. However, these deviations do not strongly affect the IR exponent which can be seen in Fig. 2(b). At 1.5T $T_{C}$ we find an IR exponent $\kappa=0.47$ and at $3 T_{C}$ we still have $\kappa=0.46$. Both results are in good agreement with the zero temperature data used in [12] for the extrapolation to the Hamiltonian limit.

In the UV, we find $\gamma=0.63$ and $m=0.22 \mathrm{GeV}$ for the $1.5 T_{C}$ propagator. Fitting with fixed $\gamma=0.5$ gives the same $\chi^{2} /$ d.o.f. and results in $m=0.44 \mathrm{GeV}$. For $3 T_{C}$ we get $\gamma=0.42$ with $m=0.83 \mathrm{GeV}$. Fixing $\gamma$ to 0.5 is not possible in this case, since $\chi^{2} /$ d.o.f. is doubled. 


\section{Coulomb potential}

The Coulomb potential in momentum space

$$
V_{C}(|\vec{p}|)=g^{2} \frac{\delta^{a b}}{N_{s}^{3}\left(N_{c}^{2}-1\right)}\left\langle\sum_{\vec{x}, \vec{y}} \mathrm{e}^{\mathrm{i} \vec{p}(\vec{x}-\vec{y})}\left[M^{-1}(-\Delta) M^{-1}\right]^{a b}(\vec{x}, \vec{y})\right\rangle
$$

is expected to behave like $V_{C}(|\vec{p}|) \sim|\vec{p}|^{-4}$ as $|\vec{p}| \rightarrow 0$, corresponding to a linear rising potential in position space at large distances. However, the extrapolation to the Coulomb string tension $\sigma_{C}$

$$
\lim _{|\vec{p}| \rightarrow 0}|\vec{p}|^{4} V_{C}(|\vec{p}|)=8 \pi \sigma_{C}
$$

is challenging for several reasons: (a) the conjugate gradient inversion of the operator is much more costly than the inversion of the ghost propagator, (b) the potential shows a large Gribov copy effect (see [7]), (c) the extrapolation is based on very few data points in the IR (see [12, 7]).

With the power of our GPU gauge fixing implementation we are able to do a detailed analysis of the Gribov copy effect on the Coulomb potential. We fixed the gauge on 5000 random copies using simulated annealing and overrelaxation. In Fig. 3(a) we compare the potential from the best

(a)

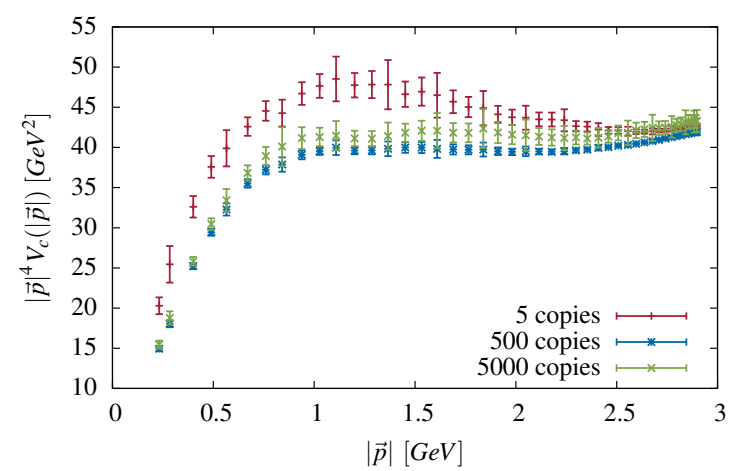

(b)

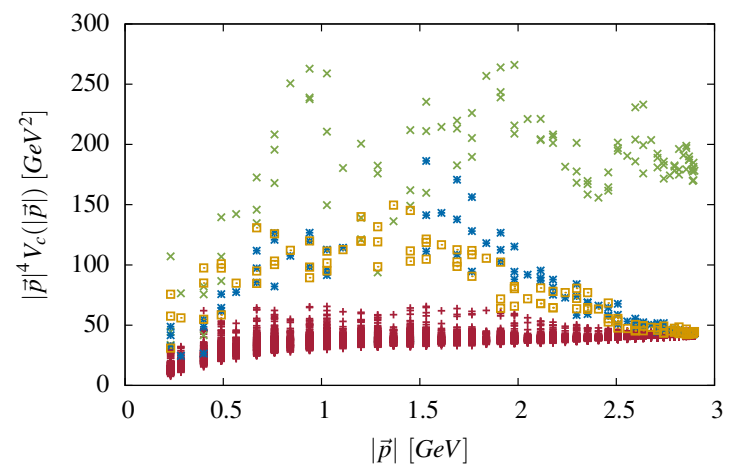

Figure 3: The Coulomb potential $|\vec{p}|^{4} V_{C}(|\vec{p}|)$ for fixed $\beta=2.2$ on a $128 \times 32^{3}$ lattice: (a) on the best gauge copy after 5, 500 and 5000 restarts for 100 configurations. (b) the raw (non-averaged) data of the best copy after 5000 restarts (corresponds to the green data points on the 1.h.s.).

copy after 5, 500 and 5000 trials for 100 configurations. Comparing 5, 100 (which we omitted in the plot) and 500 copies the result looks promising: the error bars are drastically reduced and the potential seems to converge to a stable result. However, after 5000 copies the error bars are again increased. The reason for this behaviour can be seen in Fig. 3(b) where the raw data points are plotted. The green, blue and yellow points are from three configurations which are clearly outliers compared to the red bulk of the remaining configurations. These outliers are from configurations for which the smallest eigenvalue of the operator $M(-\Delta)^{-1} M$ is more than one order of magnitude smaller compared to the smallest eigenvalues of the bulk. At the same time, the difference in the smallest eigenvalues of the Faddeev-Popov operator $M$ alone is only a factor of 2-3 and the effect on the ghost propagator from these configurations is small. To accommodate for these outliers in the statistics we need to incorporate much more configurations. 
Besides the Gribov copy effect, we are faced with huge scaling violations, see Fig. 4. In [7] the authors already found small scaling violations, though their study was for the gauge group SU(3) in a regime where discretization effects are smaller. Again, there is no difference at $1.5 T_{C}$ compared

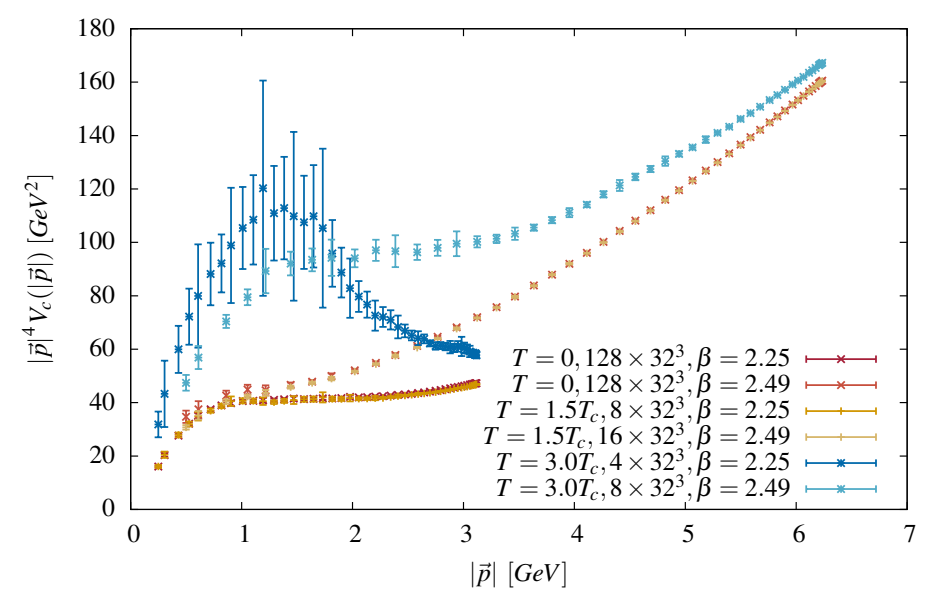

Figure 4: The Coulomb potential $|\vec{p}|^{4} V_{c}(|\vec{p}|)$ for $T=0,1.5 T_{C}$ and $3 T_{C}$.

to zero temperature, but at $3 T_{C}$ the potential changes clearly. The large error bars in the $3 T_{C}$ data is again due to one single outlier. With these problems, we are not able to conclude if at $3 T_{C}$ the Coulomb string tension changes, though very likely the string tension does not change from $T=0$ to $1.5 T_{C}$.

\section{Conclusions and outlook}

We gave results for the gluon propagator, the ghost propagator and the Coulomb potential at finite temperature. None of these quantities showed a signal of deconfinement up to $1.5 T_{C}$. We interpret this observation as an indication that a naive extension of the static Coulomb gauge propagators on the lattice to finite temperature does not work. The propagators above $T_{C}$ seem to be dominated by the rising spatial string tension and insensitive to the temporal string tension. This is also indicated by the rise in the Gribov mass of the gluon propagator.

We also found that the computation of the Coulomb potential by the definition (4.1) is hindered by several problems. Besides a strong Gribov copy effect, we find large outliers in the statistics and large scaling violations. With our current computational resources we cannot solve these problems satisfyingly.

We now investigate definitions of the Coulomb potential based on the temporal gluon propagator $\left\langle A_{0} A_{0}\right\rangle$ and partial Polyakov line correlators $[18,19]$. Whereas the former, as a quantity defined at fixed timeslice, might suffer from the same problems, the latter definition seems promising for Polyakov lines of length $\geq 2$ lattice units.

\section{Acknowledgments}

This work was partly supported by the Deutsche Forschungsgemeinschaft (DFG) under Contract No. DFG-Re856/9-1 and by the Evanglisches Studienwerk Villigst e.V. 


\section{References}

[1] C. Feuchter and H. Reinhardt, Variational solution of the Yang-Mills Schrodinger equation in Coulomb gauge, Phys.Rev. D70 (2004) 105021, [hep-th/ 0408236 ].

[2] D. Epple, H. Reinhardt, and W. Schleifenbaum, Confining Solution of the Dyson-Schwinger Equations in Coulomb Gauge, Phys.Rev. D75 (2007) 045011, [hep-th / 0612241 ].

[3] K. Langfeld and L. Moyaerts, Propagators in Coulomb gauge from SU(2) lattice gauge theory, Phys.Rev. D70 (2004) 074507, [hep-lat / 0406024 ].

[4] Y. Nakagawa, A. Nakamura, T. Saito, and H. Toki, Infrared behavior of the Faddeev-Popov operator in Coulomb gauge QCD, Phys.Rev. D75 (2007) 014508, [hep-lat/ 0702002 ].

[5] Y. Nakagawa, A. Nakamura, T. Saito, and T. Toki, The Coulomb gauge confinement scenario and the color-dependent quark potentials in lattice QCD simulations, Mod.Phys.Lett. A23 (2008) 2348-2351.

[6] Y. Nakagawa, A. Nakamura, T. Saito, and H. Toki, Confining time-like gluon and confined spatial gluons in Coulomb gauge QCD, Mod.Phys.Lett. A23 (2008) 2352-2355.

[7] A. Voigt, E.-M. Ilgenfritz, M. Muller-Preussker, and A. Sternbeck, The Effective Coulomb potential in SU(3) lattice Yang-Mills theory, Phys.Rev. D78 (2008) 014501, [arXiv: 0803.2307$].$

[8] G. Burgio, M. Quandt, and H. Reinhardt, Coulomb gauge gluon propagator and the Gribov formula, Phys.Rev.Lett. 102 (2009) 032002, [arXiv: 0807 .3291].

[9] M. Quandt, H. Reinhardt, and G. Burgio, The role of center vortices in Gribov's confinement scenario, Phys.Rev. D81 (2010) 065016, [arXiv: 1001 .3699].

[10] G. Burgio, M. Quandt, and H. Reinhardt, BRST symmetry versus Horizon condition in Yang-Mills theory, Phys.Rev. D81 (2010) 074502, [arXiv:0911.5101].

[11] Y. Nakagawa, A. Nakamura, T. Saito, and H. Toki, Scaling study of the gluon propagator in Coulomb gauge QCD on isotropic and anisotropic lattices, Phys.Rev. D83 (2011) 114503, [arXiv:1105.6185].

[12] G. Burgio, M. Quandt, and H. Reinhardt, Ghost propagator and the Coulomb form factor from the lattice, Phys.Rev. D86 (2012) 045029, [arXiv: 1205.5674 ].

[13] G. Burgio, M. Quandt, H. Reinhardt, and H. Vogt, Confinement in coulomb gauge, in proceedings of 31st International Symposium on Lattice Field Theory LATTICE 2013, POS (LATTICE 2013) 365.

[14] J. Heffner, H. Reinhardt, and D. R. Campagnari, The deconfinement phase transition in the Hamiltonian approach to Yang-Mills theory in Coulomb gauge, Phys.Rev. D85 (2012) 125029, [arXiv:1206.3936].

[15] V. Gribov, Quantization of Nonabelian Gauge Theories, Nucl.Phys. B139 (1978) 1.

[16] S. Kirkpatrick, C. Gelatt, and M. Vecchi, Optimization by Simulated Annealing, Science 220 (1983) 671-680.

[17] M. Schröck and H. Vogt, Coulomb, Landau and Maximally Abelian Gauge Fixing in Lattice QCD with Multi-GPUs, Comput.Phys.Commun. 184 (2013) 1907-1919, [arXiv: 1212.5221].

[18] E. Marinari, M. L. Paciello, G. Parisi, and B. Taglienti, The String tension in gauge theories: A Suggestion for a new measurement method, Phys.Lett. B298 (1993) 400-404, [hep-lat/9210021].

[19] J. Greensite and S. Olejnik, Coulomb energy, vortices, and confinement, Phys.Rev. D67 (2003) 094503, [hep-lat/0302018]. 